\title{
Involvement of Linker Histones in the Regulation of Replication Timing
}

\author{
Christophe Thiriet, Gwenola Auda-Boucher and Yvonnick Chéraud \\ University of Nantes/CNRS-UMR 6204 \\ Chromatin dynamics and Epigenetics
}

France

\section{Introduction}

In eukaryotic cells, genomic DNA is associated with proteins to form chromatin, wherein the basic subunit is the nucleosome (van Holde 1989; Luger et al. 1997). The histones that compose the nucleosome can undergo posttranslational modifications, which are believed to generate an epigenetic code involved in chromatin activity regulation (Jenuwein and Allis 2001). Like other chromatin activities, replication has been correlated with histone modification. However unlike other activities, such as transcription or repair, wherein core histones are specifically modified, the histone posttranslational modifications that have been shown involved in replication regulation also interest the linker histone. While the linker histone has been shown mobile within the nucleus, the way the linker histone can be associated with replication timing regulation is of general interest. The present chapter reviews structural features of chromatin and the function of linker histone in higher order of chromatin. As replication implies the accessibility of the replication machinery to DNA, the modalities that are associated with a release of compact structure involving the linker histone will be discussed as well as the function of protein kinases in this process. This will lead to a model proposing how chromatin structure can switch from a non-permissive structure to a replication competent chromatin structure. Finally, with regard to our knowledge of chromatin replication requirements and the mobility of chromatin structures, the concluding remarks point out concerns that are not yet addressed in the timely regulated process of replication.

\section{Replication of eukaryotic genomes}

Genomes of eukaryotic cells are compartmentalized within the nucleus during the interphase during which DNA is organized in chromatin. Although chromatin structure is far to be fully understood, clearly the association of proteins to DNA adds a substantial level of complexity compared to bacteria in all cellular processes that require DNA as substrate (van Holde 1989; Wolffe 1998). DNA replication does not make exception to this rule, even if it is required for faithful inheritance of the genome at each cell division and takes place only once every cell cycle. 
Despite specificities of eukaryotic genome replication, notable features of DNA duplication are shared between eukaryotes and bacteria (Mechali 2001). For instance, to be duplicated, double stranded DNA must open to make possible the access of each strand of the double helix to the DNA synthesis machineries. The initiation of the opening of DNA is performed at specific sites, named replication origins which reveal different degrees of elaboration. In Escherichia coli, DNA replication is initiated from a unique site and replication proceeds within two directions from this site. In contrast, in eukaryotes, the replication origins are multiple as it has been estimated in Chinese hamster cells that 30,000 to 50,000 origins are activated during each cell cycle (Huberman and Riggs 1966). Furthermore, among eukaryotes the actual nature and the number of replication origins are variable. Unlike higher eukaryotes, in S. cerevisiae a consensus sequence found $\sim 300$ times through the genome functions as replication origin (Nieduszynski et al. 2006). However, during the genome duplication phase of the cell cycle, not all replication origins are activated at the same time and even only a subset of the replication origins are mobilized during a considered cell cycle. The firing of replication origin is timely regulated during the S-phase. The association of DNA with proteins to form chromatin impedes the access to DNA and in a such repressive environment how DNA replication proceeds and is coordinated in space and in time across the entire genome within the living is an important question.

\subsection{The genome is structured into chromatin}

In the nucleus, the most abundant proteins associated with genomic DNA are the histone proteins. The arrangement of the histones and DNA in chromatin is highly structured and allows to the genetic information to be ordered. The packaging of DNA in eukaryotes is commonly perceived at different levels (Woodcock and Dimitrov 2001; Luger and Hansen 2005). The primary organization level of the eukaryotic genome is the nucleosome core particle. The nucleosome core particle is composed of $147 \mathrm{pb}$ DNA wrapped around the histone octamer, which contains two copies each of the four core histone proteins H2A, H2B, $\mathrm{H} 3$ and $\mathrm{H} 4$. The histones $\mathrm{H} 3$ and $\mathrm{H} 4$ form a central tetramer associated with two heterodimers of $\mathrm{H} 2 \mathrm{~A} / \mathrm{H} 2 \mathrm{~B}$ on each side composing therefore a tripartite wherein the diad axis is the symmetry axis (Arents et al. 1991; Luger et al. 1997).

Although the nucleosome core particle is defined at the atomic level and is conserved through evolution, the link between only two nucleosome core particles is more variable as the linker DNA length separating them varies between species, but also between tissues of the same organism and within a single nucleus (van Holde 1989). Furthermore, in vitro analysis of a dinucleosomal template exhibited mobility of the core histone within the template constituted of two tandem 5S RNA genes, although this sequence is known for its ability to position the nucleosome (Ura et al. 1995). Importantly, the addition of the linker histones within the dinucleosome template revealed an inhibition of nucleosome mobility (Ura et al. 1995; Ura et al. 1996). Therefore, it has been proposed that the linker histone might stabilize the nucleosomal structure by restricting the core histone mobility (Ura et al. 1995). It is clear that genome organization into a succession of nucleosomes corresponding to the beads-on-a-string results in a complex arrangement that is called higher-order chromatin structure which is still poorly understood.

Despite the striking absence of a model for higher-order chromatin structure, experiments using reconstituted nucleosomal arrays have been quite informative. Experiments analyzing the chromatin array folding showed that core histone tail domains contribute to higher- 
order formation (Tse and Hansen 1997). Similarly, core histone tail acetylation has been proposed to disrupt the higher order chromatin structure (Tse et al. 1998; Wang and Hayes 2008). In addition to the critical function of the core histones in the folding of chromatin, the linker histone has been shown to stabilize the folding of nucleosomal arrays (Carruthers et al. 1998). Indeed, extensive analyses using analytical ultracentrifugation, quantitative agarose gel electrophoresis, electron cryomicroscopy, and nuclease digestion revealed that the presence of the linker histone within nucleosomal arrays results in structures that are indistinguishable from native chicken erythrocyte chromatin (Carruthers et al. 1998).

\subsection{Linker histone acts like a genome organizer}

Although in vitro experiments using reconstituted model systems suggested an important function of linker histone (H1) in high-order chromatin structure, in vivo analyses were not as conclusive. While in the protozoan Tetrahymena the genetic depletion of the unique linker histone did not exhibit a striking phenotype (Shen et al. 1995), but a lost in transcription regulation in a gene subset and reduction in the nucleosome repeat length (Shen and Gorovsky 1996), the knock-out of this histone class in mouse cannot be achieved (Fan et al. 2003). Indeed, gene inactivation studies in the murine model exhibited a compensation effect when lacking one subtype of linker histone among the six existing in somatic cells (Fan et al. 2001). However, depletion of three subtypes led to embryonic lethality whereby developmental defects appeared as early as mid-gestation with a broad range of aberrations (Fan et al. 2003). Interestingly, similar results were observed in Drosophila suggesting that the linker histone might play a critical function in metazoans (Lu et al. 2009). Furthermore, Drosophila experiments showed that chromatin organization is impaired in absence of the linker histone and affects pericentric heterochromatin transcription ( $\mathrm{Lu}$ et al. 2009). Clearly, even if these results demonstrate a critical function of the linker histone, whether the defects appeared within individual cells missing linker histone during their lifespan or the result of epigenetic inheritance from progenitor cells remains elusive.

Surprisingly, while the linker histone exhibits a primary function in metazoan development and organization of the genome, the analyses of $\mathrm{H} 1$ binding in living cells revealed that its binding into chromatin is dynamic. Indeed, FRAP experiments using fusion linker histoneGFP revealed that following photobleaching, GFP fluorescent signal is recovered within a few minutes (Lever et al. 2000; Misteli et al. 2000). Furthermore, only minor differences in the photobleaching recovery were noticed between heterochromatin and euchromatin. However, the treatment of cells with phosphatase inhibitor, which leads to an increase of phosphorylation of the H1 C-terminal domain, resulted in a greater mobility of H1 (Lever et al. 2000). The observations of living cells provided interesting features of linker histones like their mobility in different chromatin structures. Nonetheless, whether the linker histone stability within chromatin is affected by the cell cycle stage was not addressed. Using the original methodology of incorporation of exogenous proteins within the slime mold Physarum polycephalum, allowing the analyses at specific cell cycle stages, it has been shown that the stability of linker histone binding depended upon the cell cycle stage (Thiriet and Hayes 2001). Indeed, although the efficiency of the spontaneous incorporation of exogenous linker histone was similar throughout the cell cycle, the analyses of linker histone binding to chromatin revealed a lower affinity in S-phase chromatin compared to G2-phase chromatin. Interestingly, these results suggest that chromatin activity might affect the linker histone characteristics. Noteworthy, whereas the incorporation of different linker histones revealed 
a role of different linker histone subtypes in transcription, any of the reported effects were only transiently observed (Thiriet and Hayes 1999). It has been shown that exogenous core histones incorporated into Physarum were stable throughout the cell cycle (Prior et al. 1980; Prior et al. 1983; Thiriet and Hayes 2005), the instability of linker histones in this organism points out the issue of the half life of cellular linker histones. Indeed, to date we note a critical absence of studies determining the half-life of histone proteins.

\subsection{Learning linker histone mechanism from transcription}

Genetic depletion of linker histones in the unicellular Tetrahymena did not exhibit a striking phenotype of the cells, although nuclear volume was enlarged and evidenced the involvement of linker histones in chromatin packaging (Shen et al. 1995). Despite the global structural effect of linker histones in the folding of genetic information, chromatin activity analyses of the Tetrahymena H1 knocked-out strain showed effects on transcriptional activities both positively and negatively of specific genes (Shen and Gorovsky 1996). Importantly, the specific transcription profiles determined within the knocked-out strain was recapitulated in the strain wherein linker histone phosphorylation sites were mutated to glutamic acid, mimicking the fully phosphorylated state of the histone (Dou et al. 1999). Further investigations of the mechanism by which linker histone phosphorylation affects transcription activity revealed the generation of mutant strains wherein the charge resembled that of the phosphorylated state without mimicking the structure of the phosphorylation induced transcription defects (Dou and Gorovsky 2000). Therefore, it was concluded that $\mathrm{H} 1$ phosphorylation acts by changing the overall charge within the histone domain, rather than by direct recognition of the phosphate added by the post-translational modification.

The potential lack of physical recognition of the added phosphate in the carboxy-terminal domain of $\mathrm{H} 1$ associated with transcription is consistent with the idea that this unstructured domain of the linker histone is intrinsically disordered (Hansen et al. 2006). Unlike other histone classes, the linker histones comprise a family presenting variability between members. Interestingly, six isoforms of $\mathrm{H} 1$ have been identified in most higher eukaryotes, and several isoforms can localize within a single cell (Alami et al. 2003). Although the actual function of the variability of linker histones is undetermined, most linker histones share an identical structure composed of an unstructured amino-terminal domain, a globular domain defined by a three a-helix and an unstructured carboxy-terminal domain that can be subjected to phosphorylation. Conversely, the amino-acid composition of the carboxyterminal domain of linker histones is amazingly similar between isoforms, although the sequences diverge. These remarkable properties led to propose that the carboxy-terminal domain of linker histones might function as an intrinsically disordered region, wherein the global amino-acid composition rather than the actual primary sequence would provide the chromatin binding properties (Hansen et al. 2006).

\subsection{Linker histone function in replication}

In contrast to transcription, replication of the genome takes place only once per cell cycle during the S-phase. The infrequency of the replication activity at determined genome location significantly complicates chromatin replication mechanisms. This experimental difficulty can be over-ruled using systems that exhibited synchronous nuclear activities either induced artificially with blocking reagents followed by cell released, or with cellular 
models exhibiting naturally synchronous activities within a population of nuclei. The powerful model system Physarum polycephalum enables to examine chromatin replication mechanisms, as at the plasmodial stage of the life cycle of this organism grows by successive cell cycles and forms a syncytium with a large of nuclei (estimated to $\sim 510^{8}$ in a usually used $5-7 \mathrm{~cm}$ diameter macroplasmodium) in a unique cytoplasm conferring to the nucleus population a perfect synchrony (Thiriet and Hayes 1999). These specific characteristics have been useful for performing analyses of replication using biochemical approaches, such as the determination of the replication timing of specific genes during S-phase, the mapping of replication origins in absence of a consensus sequence and recently the relationship between chromatin structure and replication timing (Thiriet and Hayes 2009).

It has been shown that the incorporation of exogenous linker histones was stably associated with chromatin only in the G2-phase and exhibit significant inhibition of transcription in correlation with the linker histone subtype that was introduced into the cell (Thiriet and Hayes 2001). This inhibitory effect of the linker histone seemed controversial with the absence of global effect of linker histones observed in Tetrahymena (Shen and Gorovsky 1996). Nevertheless, it is important to note that the experimental designs in both analyses were somehow opposite as genetic depletion was carried out in Tetrahymena while in the Physarum experiments additional linker histones were added. Therefore, it was of special interest to examine the effects of linker histone depletion in the Physarum model system. This was achieved by knocking-down the expression of linker histones (Thiriet and Hayes 2009). Interestingly, as the nuclei are perfectly synchronous throughout the cell cycle, siRNA can be incorporated and analyzed at specific cell cycle stages. Unexpectedly, the observation of Physarum cells revealed a faster cellular growth in the early S-phase under linker histone depletion. The cell cycle stage specificity of the $\mathrm{H} 1$ depletion led to determine whether replication was affected by the absence of linker histones. By carrying out pulses of incorporation of radiolabelled DNA precursor during the duration of the S-phase followed by the determination of specific activity of the genomic DNA, it was observed that the maximum of radioactivity was reached faster in H1-depleted cells than in controls. Importantly, as the maximum of radioelement contained in DNA was similar in both experimental conditions and reached a plateau corresponding to replication completion, the genome was thus duplicated only once in presence and in absence of the linker histones. Therefore, the linker histones did not initiate multiple rounds of replication of chromatin regions, but affect the rate of chromatin duplication.

While these experiments revealed a global function in the control of the ubiquitous activity of genome replication, consistently with the deleterious effects of partial depletion in metazoan development, the mechanism by which $\mathrm{H} 1$ acts on replication needed to be clarified. Indeed, two distinct mechanisms could account for the acceleration of genome duplication. Following initiation, the fork of replication might progress faster through chromatin. Alternatively, the linker histone might directly act on the firing of replication origins. It has been shown in metazoans that the setting up of replication origins is performed by a multi-step process prior to the cell cycle dedicated to genome replication. These steps should be tightly controlled as only a subset of the potential replication origins are activated at each cell cycle and any origin is activated only once in the S-phase. Therefore, labelling of the replication origins is required and this involves their recognition by factors that associate with DNA to form a pre-replication complex wherein the upgrade 
will raise to the initiation complex. Throughout the S-phase, the initiation complex is temporally coordinated for firing at specific times during the duplication stage of the cell cycle (Maric and Prioleau 2010). The choice of the timing of the replication origin firing is not harmless for the cell, as replication timing has been correlated with the transcriptional activity of genes. Unambiguous demonstration of this correlation was performed in Physarum, wherein two distinct copies of the developmentally regulated genes encoding for profilin exhibited a reprogramming of their timing of replication linked to transcriptional activity specific to each profiling gene (Maric et al. 2003). Consistently, analyses in mouse embryonic stem cells exhibited changes in the replication program during cell differentiation (Hiratani et al. 2008).

The molecular mechanism leading to faster replication in conjunction with the absence of linker histone was elucidated by pulse labelling experiments of replicating chromatin with a thymidine analogue. Microscopic observations of the incorporation of the analogue into genomic DNA revealed that the number of distinguishable foci almost double in absence of $\mathrm{H} 1$, whereas the intensity of the foci which reflected the amount of incorporated precursor remains statistically unchanged (Thiriet and Hayes 2009). It was therefore concluded that depletion of linker histones has merely disturbed replication timing regulation and not the velocity of the replication fork progression through chromatin. These results were in agreement with the determination of the replication timing of specific replicons. The early establishment of the usage of the replication origins and the temporal coordination that is associated to their activation suggested the existence of an epigenetic control. Remarkably, the abolishment of the replication epigenetic control coincides with the depletion of linker histones. It is therefore reasonable to propose that linker histones are involved in the epigenetic regulation of chromatin replication.

\subsection{Epigenetic repression abolishment by $\mathrm{H} 1$ phosphorylation}

The studies of $\mathrm{H} 1$ function during transcription showed that the mimics of $\mathrm{H} 1$ phosphorylation exhibit transcription defects closely related to genetic depletion of the linker histone (Dou et al. 1999; Dou and Gorovsky 2000). Accordingly, it was proposed that the phosphorylation of $\mathrm{H} 1$ facilitates the mobility of the linker histone (Lever et al. 2000). Hence, to verify whether this post-translational modification of $\mathrm{H} 1$ might also affect replication, inhibition of phosphatase activity was performed and replication effects were determined. The analysis of a lately replicated locus revealed incorporation of thymidine analogue in early S-phase concomitantly with hyperphosphorylation of the linker histone (Thiriet and Hayes 2009). Although H1 has been shown in vitro to be an excellent substrate for many kinases (Ducommun et al. 1990), the complex containing the kinase Cdk2 and Cdc45 displays the characteristics of a good candidate to accomplish this task in S-phase. Indeed, transfection of Cdc45 promotes chromatin decondensation and co-localized phosphorylated $\mathrm{H} 1$ in culture (Alexandrow and Hamlin 2005). Co-immunoprecipitation experiments revealed the formation of a complex containing at least Cdc45 and Cdk2. Furthermore, determination of the sequential deposition to chromatin revealed that Cdc45 associates with chromatin prior to $\mathrm{Cdk} 2$ suggesting that $\mathrm{Cdc} 45$ recruits $\mathrm{Cdk} 2$ to chromatin targets. Interestingly, the same study showed that the Cyclin A kinase associated with chromatin with kinetics nearly identical to those of Cdk2, and suggested that the H1 kinase activity might be redundant in the S-phase (Alexandrow and Hamlin 2005). 


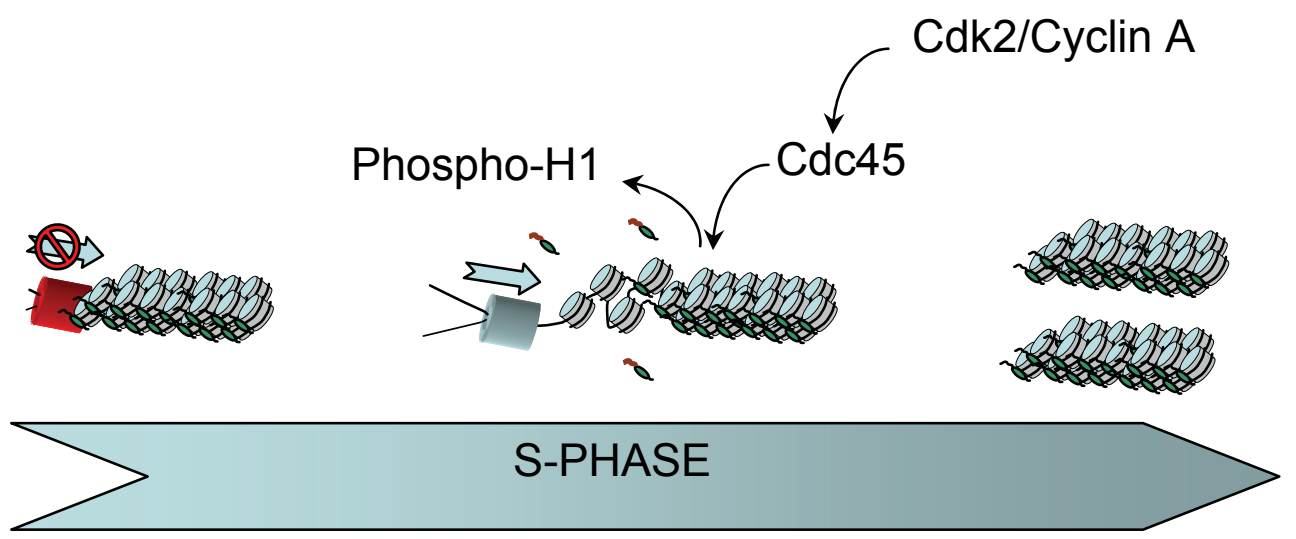

Fig. 1. Model of replication origin firing induced by $\mathrm{H} 1$ phosphorylation. The repressive chromatin structure for replication proceeding (left) becomes permissive after the release of the linker histone induced by $\mathrm{H} 1$ phosphorylation (middle) and leads the duplication of chromatin (right).

\section{Conclusion}

Despite the ubiquitous composition of chromatin, among histone classes, the linker histone presents the greatest variability through evolution and between subtypes from a single organism. On the basis of in vitro analyses of linker histones, their function has been associated with chromatin folding and higher order structure. However, the biochemical features that are common to all linker histone subtypes, do not provide satisfactory explanations to the embryonic lethality observed in mouse when three from the six somatic isoforms are depleted, whereas the depletion of only one isoform exhibits compensation effects (Fan et al. 2001). Therefore, understanding the biological function of linker histones within eukaryotic cells is a major task. Surprisingly, while metazoans showed essential roles of linker histones in early development, the lack of $\mathrm{H} 1$ in protozoans did not exhibit drastic phenotypes and was even depicted like a transcription regulator in a subset of genes (Fan et al. 2003). One issue in these observations was the rational between the contrasted effects of H1. It was unlikely that the result of evolution was to generate divergent function with no alteration of biochemical properties. Thus, the linker histone function possibly required to act on a global chromatin activity that needs to be tightly coordinated during development. Unexpectedly, it was shown in the slime mold Physarum polycephalum that cells lacking linker histone exhibited a lost in the regulation of the replication origin firing, which was also associated with an increase of DNA accessibility (Thiriet and Hayes 2009). These experiments led to propose that linker histones might have a critical role in replication timing regulation (Fig.1). Although these experiments were the first demonstration of a global effect of linker histones, they are consistent with the genome regulation requirement observed during development and differentiation. Nonetheless, if linker histone function has been proposed to temporally regulation replication of chromatin, the issue of variety of the linker histone isoforms is not yet addressed in the replication context. 


\section{Acknowledgements}

We are grateful to members of the Thiriet's lab for valuable discussions. Our group is funded by "La ligue contre le cancer" (Committees 41, 44 and 86), ANR, Cancéropôle Grand-Ouest, CNRS, and University of Nantes.

\section{References}

Alami, R., Y. Fan, S. Pack, T.M. Sonbuchner, A. Besse, Q. Lin, J.M. Greally, A.I. Skoultchi, and E.E. Bouhassira. 2003. Mammalian linker-histone subtypes differentially affect gene expression in vivo. Proc Natl Acad Sci U S A 100: 5920-5.

Alexandrow, M.G. and J.L. Hamlin. 2005. Chromatin decondensation in S-phase involves recruitment of Cdk2 by Cdc45 and histone H1 phosphorylation. J Cell Biol 168: 87586.

Arents, G., R.W. Burlingame, B.C. Wang, W.E. Love, and E.N. Moudrianakis. 1991. The nucleosomal core histone octamer at 3.1 A resolution: a tripartite protein assembly and a left-handed superhelix. Proc Natl Acad Sci U S A 88: 10148-52.

Carruthers, L.M., J. Bednar, C.L. Woodcock, and J.C. Hansen. 1998. Linker histones stabilize the intrinsic salt-dependent folding of nucleosomal arrays: mechanistic ramifications for higher-order chromatin folding. Biochemistry 37: 14776-87.

Dou, Y. and M.A. Gorovsky. 2000. Phosphorylation of linker histone H1 regulates gene expression in vivo by creating a charge patch. Mol Cell 6: 225-31.

Dou, Y., C.A. Mizzen, M. Abrams, C.D. Allis, and M.A. Gorovsky. 1999. Phosphorylation of linker histone $\mathrm{H} 1$ regulates gene expression in vivo by mimicking $\mathrm{H} 1$ removal. Mol Cell 4: 641-7.

Ducommun, B., Y. Tollon, M. Gares, D. Beach, and M. Wright. 1990. Cell cycle regulation of p34cdc2 kinase activity in Physarum polycephalum. J Cell Sci 96 ( Pt 4): 683-9.

Fan, Y., T. Nikitina, E.M. Morin-Kensicki, J. Zhao, T.R. Magnuson, C.L. Woodcock, and A.I. Skoultchi. 2003. H1 linker histones are essential for mouse development and affect nucleosome spacing in vivo. Mol Cell Biol 23: 4559-72.

Fan, Y., A. Sirotkin, R.G. Russell, J. Ayala, and A.I. Skoultchi. 2001. Individual somatic H1 subtypes are dispensable for mouse development even in mice lacking the H1(0) replacement subtype. Mol Cell Biol 21: 7933-43.

Hansen, J.C., X. Lu, E.D. Ross, and R.W. Woody. 2006. Intrinsic protein disorder, amino acid composition, and histone terminal domains. J Biol Chem 281: 1853-6.

Hiratani, I., T. Ryba, M. Itoh, T. Yokochi, M. Schwaiger, C.W. Chang, Y. Lyou, T.M. Townes, D. Schubeler, and D.M. Gilbert. 2008. Global reorganization of replication domains during embryonic stem cell differentiation. PLoS Biol 6: e245.

Huberman, J.A. and A.D. Riggs. 1966. Autoradiography of chromosomal DNA fibers from Chinese hamster cells. Proc Natl Acad Sci U S A 55: 599-606.

Jenuwein, T. and C.D. Allis. 2001. Translating the histone code. Science 293: 1074-80.

Lever, M.A., J.P. Th'ng, X. Sun, and M.J. Hendzel. 2000. Rapid exchange of histone H1.1 on chromatin in living human cells. Nature 408: 873-6.

Lu, X., S.N. Wontakal, A.V. Emelyanov, P. Morcillo, A.Y. Konev, D.V. Fyodorov, and A.I. Skoultchi. 2009. Linker histone H1 is essential for Drosophila development, the establishment of pericentric heterochromatin, and a normal polytene chromosome structure. Genes Dev 23: 452-65. 
Luger, K. and J.C. Hansen. 2005. Nucleosome and chromatin fiber dynamics. Curr Opin Struct Biol 15: 188-96.

Luger, K., A.W. Mader, R.K. Richmond, D.F. Sargent, and T.J. Richmond. 1997. Crystal structure of the nucleosome core particle at 2.8 A resolution. Nature 389: 251-60.

Maric, C., M. Benard, and G. Pierron. 2003. Developmentally regulated usage of Physarum DNA replication origins. EMBO Rep 4: 474-8.

Maric, C. and M.N. Prioleau. 2010. Interplay between DNA replication and gene expression: a harmonious coexistence. Curr Opin Cell Biol 22: 277-83.

Mechali, M. 2001. DNA replication origins: from sequence specificity to epigenetics. Nat Rev Genet 2: 640-5.

Misteli, T., A. Gunjan, R. Hock, M. Bustin, and D.T. Brown. 2000. Dynamic binding of histone H1 to chromatin in living cells. Nature 408: 877-81.

Nieduszynski, C.A., Y. Knox, and A.D. Donaldson. 2006. Genome-wide identification of replication origins in yeast by comparative genomics. Genes Dev 20: 1874-9.

Prior, C.P., C.R. Cantor, E.M. Johnson, and V.G. Allfrey. 1980. Incorporation of exogenous pyrene-labeled histone into Physarum chromatin: a system for studying changes in nucleosomes assembled in vivo. Cell 20: 597-608.

Prior, C.P., C.R. Cantor, E.M. Johnson, V.C. Littau, and V.G. Allfrey. 1983. Reversible changes in nucleosome structure and histone $\mathrm{H} 3$ accessibility in transcriptionally active and inactive states of rDNA chromatin. Cell 34: 1033-42.

Shen, X. and M.A. Gorovsky. 1996. Linker histone H1 regulates specific gene expression but not global transcription in vivo. Cell 86: 475-83.

Shen, X., L. Yu, J.W. Weir, and M.A. Gorovsky. 1995. Linker histones are not essential and affect chromatin condensation in vivo. Cell 82: 47-56.

Thiriet, C. and J.J. Hayes. 1999. Histone proteins in vivo: cell-cycle-dependent physiological effects of exogenous linker histones incorporated into Physarum polycephalum. Methods 17: 140-50.

-. 2001. Assembly into chromatin and subtype-specific transcriptional effects of exogenous linker histones directly introduced into a living Physarum cell. J Cell Sci 114: 965-73.

-. 2005. Replication-independent core histone dynamics at transcriptionally active loci in vivo. Genes Dev 19: 677-82.

-. 2009. Linker histone phosphorylation regulates global timing of replication origin firing. J Biol Chem 284: 2823-9.

Tse, C. and J.C. Hansen. 1997. Hybrid trypsinized nucleosomal arrays: identification of multiple functional roles of the $\mathrm{H} 2 \mathrm{~A} / \mathrm{H} 2 \mathrm{~B}$ and $\mathrm{H} 3 / \mathrm{H} 4 \mathrm{~N}$-termini in chromatin fiber compaction. Biochemistry 36: 11381-8.

Tse, C., T. Sera, A.P. Wolffe, and J.C. Hansen. 1998. Disruption of higher-order folding by core histone acetylation dramatically enhances transcription of nucleosomal arrays by RNA polymerase III. Mol Cell Biol 18: 4629-38.

Ura, K., J.J. Hayes, and A.P. Wolffe. 1995. A positive role for nucleosome mobility in the transcriptional activity of chromatin templates: restriction by linker histones. Embo J 14: 3752-65.

Ura, K., K. Nightingale, and A.P. Wolffe. 1996. Differential association of HMG1 and linker histones B4 and $\mathrm{H} 1$ with dinucleosomal DNA: structural transitions and transcriptional repression. Embo J 15: 4959-69.

van Holde, K.E. 1989. Chromatin. Springer-Verlag, New-York. 
Wang, X. and J.J. Hayes. 2008. Acetylation mimics within individual core histone tail domains indicate distinct roles in regulating the stability of higher-order chromatin structure. Mol Cell Biol 28: 227-36.

Wolffe, A.P. 1998. Chromatin: Structure and Function. Academic Press, San Diego.

Woodcock, C.L. and S. Dimitrov. 2001. Higher-order structure of chromatin and chromosomes. Curr Opin Genet Dev 11: 130-5. 


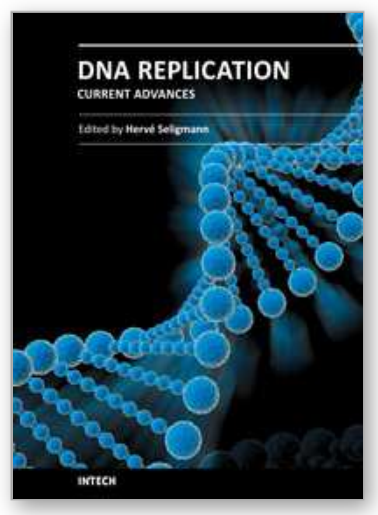

\section{DNA Replication-Current Advances}

Edited by Dr Herve Seligmann

ISBN 978-953-307-593-8

Hard cover, 694 pages

Publisher InTech

Published online 01, August, 2011

Published in print edition August, 2011

The study of DNA advanced human knowledge in a way comparable to the major theories in physics, surpassed only by discoveries such as fire or the number zero. However, it also created conceptual shortcuts, beliefs and misunderstandings that obscure the natural phenomena, hindering its better understanding. The deep conviction that no human knowledge is perfect, but only perfectible, should function as a fair safeguard against scientific dogmatism and enable open discussion. With this aim, this book will offer to its readers 30 chapters on current trends in the field of DNA replication. As several contributions in this book show, the study of DNA will continue for a while to be a leading front of scientific activities.

\section{How to reference}

In order to correctly reference this scholarly work, feel free to copy and paste the following:

Christophe Thiriet, Gwenola Auda-Boucher and Yvonnick Chéraud (2011). Involvement of Linker Histones in the Regulation of Replication Timing, DNA Replication-Current Advances, Dr Herve Seligmann (Ed.), ISBN: 978-953-307-593-8, InTech, Available from: http://www.intechopen.com/books/dna-replication-currentadvances/nvolvement-of-linker-histones-in-the-regulation-of-replication-timing

\section{INTECH}

open science | open minds

\section{InTech Europe}

University Campus STeP Ri

Slavka Krautzeka 83/A

51000 Rijeka, Croatia

Phone: +385 (51) 770447

Fax: +385 (51) 686166

www.intechopen.com

\section{InTech China}

Unit 405, Office Block, Hotel Equatorial Shanghai

No.65, Yan An Road (West), Shanghai, 200040, China 中国上海市延安西路65号上海国际贵都大饭店办公楼 405 单元

Phone: $+86-21-62489820$

Fax: +86-21-62489821 
(C) 2011 The Author(s). Licensee IntechOpen. This chapter is distributed under the terms of the Creative Commons Attribution-NonCommercialShareAlike-3.0 License, which permits use, distribution and reproduction for non-commercial purposes, provided the original is properly cited and derivative works building on this content are distributed under the same license. 